\title{
The reciprocal relationship between coping mechanisms and lung cancer diagnosis: findings of a prospective study
}

This article was published in the following Dove Press journal: Cancer Management and Research

\author{
Cristian Oancea' \\ Cristian Suciu ${ }^{2}$ \\ Bogdan Timar ${ }^{3}$ \\ Ion Papava ${ }^{4}$ \\ Marius Raica ${ }^{2}$ \\ Ovidiu Burlacu ${ }^{5}$ \\ 'Department of Pulmonology, \\ ${ }^{2}$ Department of Microscopic \\ Morphology/Histology, ${ }^{3}$ Department \\ of Biostatistics and Medical \\ Informatics, ${ }^{4}$ Department of \\ Neurosciences, ${ }^{5}$ Department of \\ Surgical Semiology, "Victor Babes" \\ University of Medicine and Pharmacy, \\ Timisoara, Romania
}

Background/purpose: Lung cancer is a major stress factor for the affected individual, leading to psychological distress in over $50 \%$ of the diagnosed patients. Since coping styles describe different patterns in approaching serious problems, our study aimed at ascertaining if the diagnosis of lung cancer has an impact on the patient's coping styles and if there is a difference in psychical response among patients with different coping styles, as assessed by variance of anxiety and depression scores after diagnosis.

Patients and methods: In this prospective study, a cohort of 50 patients were evaluated using the COPE scale, Generalized Anxiety Disorder Questionnaire 7 (GAD-7), and Patient Health Questionnaire-9 (PHQ-9), both prior to and 1 month after learning about their lung cancer diagnosis. The baseline and the final parameters were compared and stratified with respect to coping styles. Results: We observed that 1 month after learning the diagnosis, the patients had a significantly higher GAD-7 score (median score 12 vs 4 points; $p<0.001$ ). At the same time, the PHQ-9 score was significantly higher at the 1 month follow-up time-point (median score 16 vs $7 ; p=0.002$ ). The increases in the anxiety scores were significant in patients with initial social support (13 vs $3 ; p=0.014$ ) and avoidance coping style ( 14 vs $6 ; p=0.003$ ). Regarding the depression scores, after the diagnosis, the only significant increase was observed in patients with initial avoidance coping style ( 18 vs $5 ; p=0.014$ ).

Conclusion: Our study demonstrates that patients who receive the diagnosis for cancer show a significant increase in anxiety and depression intensity. The most adaptive coping style turned out to be the problem-focused one while the least adaptive one was the avoidant style.

Keywords: lung cancer, anxiety, depression, behavior, psychosocial, distress

\section{Introduction}

Lung cancer is a serious condition with often limited life expectancy despite medical advances. ${ }^{1}$ It is one of the most common types of cancer affecting both men and women. ${ }^{2}$ Lung cancer is the second most commonly diagnosed cancer in both men and women in the United States. An estimated 222,500 new cases of lung cancer will be diagnosed in 2017 , accounting for about $25 \%$ of all cancer diagnoses. ${ }^{3}$ Cancer is a major stress and traumatic factor for every individual, leading to psychological distress in over $50 \%$ of the patients. ${ }^{4-6}$

Thus, the symptoms of anxiety and depression are frequent in patients diagnosed with lung cancer. Previous studies mention the presence of significant clinical levels of anxiety and depression in over $52 \%$ of subjects with cancer. ${ }^{7,8}$ Treating these psychiatric comorbidities in patients with lung cancer needs to be a priority because they influence the quality of life, adherence to treatment, and mortality. ${ }^{6,-11}$
Correspondence: Cristian Suciu Department of Microscopic Morphology/ Histology, "Victor Babes" University of Medicine and Pharmacy, Eftimie Murgu Squ number 2, 3004I Timișoara, Romania Tel +40 745393344

Email cristian_suciu@umft.ro 
Anxiety and depression also depend on the coping ability of the patients. ${ }^{12-14}$ The coping mechanism represents a psychological process that takes place in the conscious plan of the psychism that we use to manage the difficult and stressful situations in our daily life. Although there are extensive studies in literature regarding coping in cancer, few relate to coping in patients with lung cancer. ${ }^{15}$

There are individuals who in stress situations use coping mechanisms that lead to a positive psychological response such as personal enrichment and greater appreciation. ${ }^{16}$ The personal coping style although generally characterized by a certain stability over time can vary as a result of the coping mechanisms that the subject has to mobilize when faced with more particular stressful life situations, such as cancer. The majority of the studies that approached coping in patients with cancer were cross-sectional and few addressed its dynamic aspect. Thus, the specific coping mechanism was questionable because most of the information came from cross-sectional studies. ${ }^{17,18}$

We consider that the coping style represents an important psychological dimension that has to be taken into account in the therapeutic approach of the patient with lung cancer. Hence, we aimed to analyze if the diagnosis of lung cancer has an impact on the patients' coping styles and if there is a difference in psychical response among patients with different coping styles, as assessed by variance of anxiety and depression scores after the diagnosis.

\section{Patients and methods}

\section{Patients}

In this prospective study, a cohort of 50 patients with lung cancer were analyzed. All patients signed an informed consent form to participate in the study prior to any study procedures. The research was approved by the ethical board of the Clinical Hospital of Infectious Diseases and Pneumology in Timisoara. The patients' demographic characteristics are presented in Table 1.

Table I Patients' demographic characteristics

\begin{tabular}{ll}
\hline Parameter & Value \\
\hline Male gender, n (\%) & $36(72.0 \%)$ \\
Married individuals, n (\%) & $39(78.0 \%)$ \\
Education & \\
$\quad$ Primary education, n (\%) & $6(12 \%)$ \\
$\quad$ Medium education - high school, n (\%) & $11(22 \%)$ \\
$\quad$ Superior education - university degree, n (\%) & $33(66 \%)$ \\
Age (years), median (interquartile distance) & $60(6)$ \\
Body mass index (average, standard deviation) & $20.9 \pm 2.3$ \\
Smoking years, median (interquartile distance) & $21.5(15)$ \\
\hline
\end{tabular}

\section{Methods}

Patient selection included individuals who came for control of the presenting pulmonary symptoms and who were suspicious for lung cancer. After receiving the results from the lung biopsy, we included only patients with confirmed lung cancer in the study. Before receiving the results, the patients completed the questionnaires. After 1 month, they completed the questionnaires again. The questionnaires were: COPE scale, Generalized Anxiety Disorder Questionnaire 7 (GAD-7) and Patient Health Questionnaire-9 (PHQ-9). The study was conducted in the period February 10, 2016 to January 102017.

The COPE scale inventory is a self-administered questionnaire, which evaluates coping strategies that individuals use to manage stressful situations in life. ${ }^{19}$ The scale comprises 60 questions with answers rated from 1 to 4 , where 1 means "I usually don't do this thing" and 4 means "I often do this thing." The coping ways are represented by the four coping styles that result from enlisting the following coping mechanisms: 1) problem-focused coping, which includes planning, active approach, and deletion of concurrent activities; 2) emotion-focused coping, which includes positive interpretation and growth, restraint, and acceptance; 3) social support-focused coping, which includes the use of social-instrumental support, the use of the social-emotional support, and the expression of feelings (venting of emotions); 4) avoidant coping, which includes denial, mental, and behavioral deactivation. The highest score of these four coping styles indicates the more frequent use of it. ${ }^{20}$

The PHQ-9 is a self-reported questionnaire that is used to evaluate the presence and severity of depression. PHQ-9 test consists of nine items that are based directly on the nine diagnostic criteria for major depressive disorders in the Diagnostic and Statistical Manual of Mental Discorders, Fourth Edition. It can estimate depressive distress as well as grade of depressive symptom severity. A higher PHQ-9 score is associated with more severe depression; based on this score, the severity of depression may be divided into three groups: minimal or mild (PHQ-9 score $<10$ ), moderate (PHQ-9 score 10-19), and severe (PHQ-9 score $>19){ }^{21}$

GAD-7 is a self-reported questionnaire, which measures severity of anxiety. GAD-7 has seven items, which measure severity of various signs of GAD according to reported response categories with assigned points. Assessment is indicated by the total score, which results by adding together the scores of the scales of all the seven items. GAD-7 total score for the seven items ranges from 0 to 21 . Interpretation: scores of 5, 10, and 15 represent cutoff points for mild, moderate, and severe anxiety. ${ }^{22}$ 


\section{Statistical analysis}

Data were collected and analyzed using the SPSS v.17 software suite (SPSS Inc. Chicago, IL, USA) and are presented as medians and interquartile range for continuous variables without Gaussian distribution, average \pm standard deviation for continuous variables with Gaussian distribution, or number of individuals and percentage from the subgroup total in the case of nominal variables.

Prior enrollment sample size estimation was performed using data from literature (variations, standard deviations, and minimum detectable difference). Based on this calculation, a number of 50 individuals provided a statistical power of $80 \%$ and a corresponding $\alpha$ of 0.05 in the case of no missing data, withdrawals, or loss to follow-up.

The normality of the distribution of variables was tested prior to analysis according to the method described by Kolmogorov and Smirnoff (in this test, a $p$-value $<0.05$ was considered to be associated with a non-Gaussian value distribution).

To assess the significance of the differences among groups (problem-focused coping, emotion-focused coping, social support-focused coping, and avoidance coping), MannWhitney $U$, Kruskal-Wallis (medians, non-Gaussian populations), and chi-square (percentages) tests were performed. The correlation between studied variables was evaluated using Spearman's rank sum correlation coefficient (nonGaussian distributed variables), its statistical significance being assessed using the $t$-distribution score test. In this study, a $p$-value $<0.05$ was considered as the threshold for statistical significance.

\section{Results}

\section{The impact of diagnosis on anxiety and depression}

We observed that 1 month after receiving the diagnosis of their disease, the patients had a significantly higher GAD-7 score (median score 12 vs 4 points; $p<0.001$ ), having the median of paired differences of 4 points. The differences are presented in Table 2 and Figure 1.

At the same time, the PHQ-9 score was significantly higher at 1 month follow-up time-point (median score 16 vs $7 ; p=0.002$ ), with a median of paired differences of 2 points. The comparative results are presented in Table 3 and Figure 2.

We observed that the most important increases in the GAD-7 score (Figure 3) were present in patients with initial avoidance coping (median increase with 7 points at the follow-up), followed by patients with initial social supportfocused coping (median increase, 3 points), emotion-focused coping (median increase, 2 points), and problem-focused coping (median increase, with 1 point). The differences regarding the modification of GAD-7 score were statistically significant $(p=0.030)$.

Regarding the PHQ-9 score (Figure 4), patients with initial avoidance coping had the most important increase in score (median increase, 13 points) followed by patients with initial emotion-focused coping (median increase with 2 points). Patients with initial problem-focused coping and those with

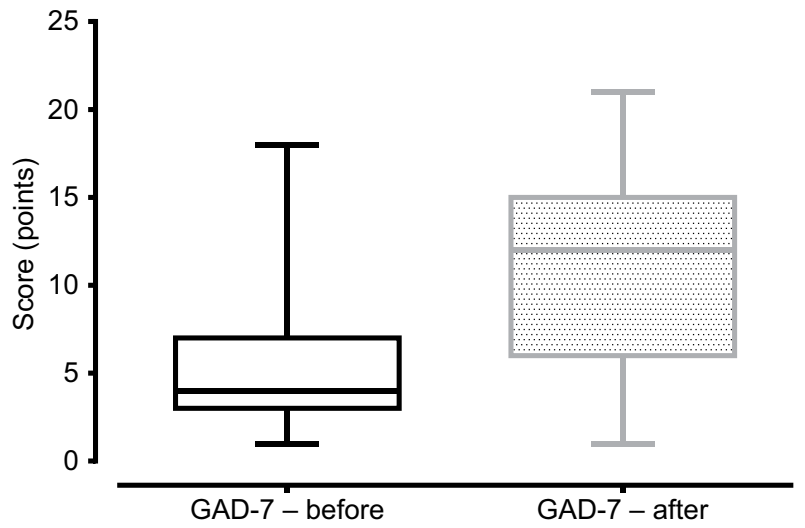

Figure I Box and whiskers plot comparison for GAD-7 scores before and after diagnosis.

Abbreviation: GAD-7, Generalized Anxiety Disorder 7.

Table 2 Comparison of GAD-7 score before and after the diagnosis

\begin{tabular}{llll}
\hline & GAD-7 - before diagnosis & GAD-7 - after diagnosis & GAD-7 score after-before \\
\hline Minimum & 1.000 & 1.000 & -6.000 \\
25 Percentile & 3.000 & 6.000 & 0.0 \\
Median & 4.000 & 12.00 & 4.000 \\
75 Percentile & 7.000 & 15.00 & 11.00 \\
Maximum & 18.00 & 21.00 & 15.00 \\
Mean & 5.936 & 10.79 & $4.85 \mathrm{I}$ \\
Standard deviation & 4.474 & 5.618 & 6.266 \\
\hline
\end{tabular}

Abbreviation: GAD-7, Generalized Anxiety Disorder 7. 
Table 3 Comparison of PHQ-9 score before and after diagnosis

\begin{tabular}{llll}
\hline & PHQ-9 before diagnosis & PHQ-9 after diagnosis & PHQ-9 score after-before \\
\hline Minimum & 2.000 & 2.000 & -11.00 \\
25 Percentile & 4.000 & 10.00 & -1.000 \\
Median & 7.000 & 16.00 & 2.000 \\
75 Percentile & 14.00 & 18.00 & 14.00 \\
Maximum & 23.00 & 25.00 & 18.00 \\
Mean & 9.298 & 14.21 & 4.915 \\
Standard deviation & 6.213 & 6.199 & 8.121 \\
\hline
\end{tabular}

Abbreviation: PHQ-9, Patient Health Questionnaire-9.

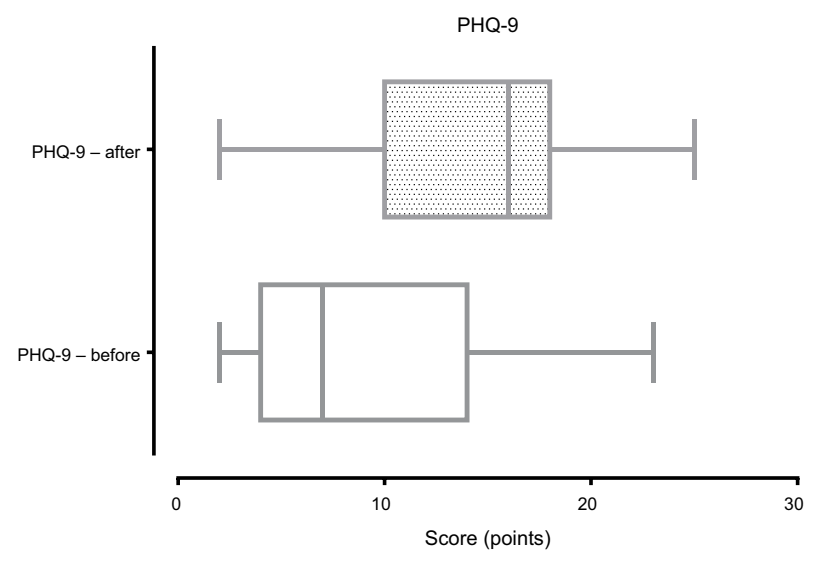

Figure 2 Box and whiskers plot comparison for PHQ-9 scores before and after diagnosis.

Abbreviation: PHQ-9, Patient Health Questionnaire-9.

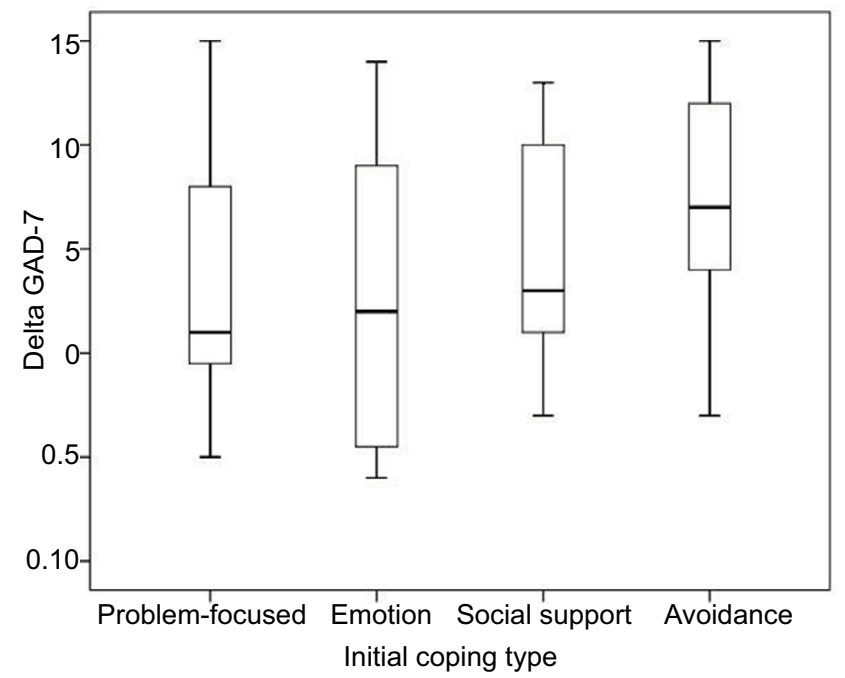

Figure 3 Variation of GAD-7 scores according to initial patients' coping type. Abbreviation: GAD-7, Generalized Anxiety Disorder 7.

social support-focused coping had the same median PHQ-9 score before and after finding out about their diagnosis.

The variations in GAD-7 and PHQ-9 scores according to initial patients' coping type are detailed in Table 4.

We observed that patients with initial avoidance coping style had the highest GAD-7 (median 14 points) and PHQ-9

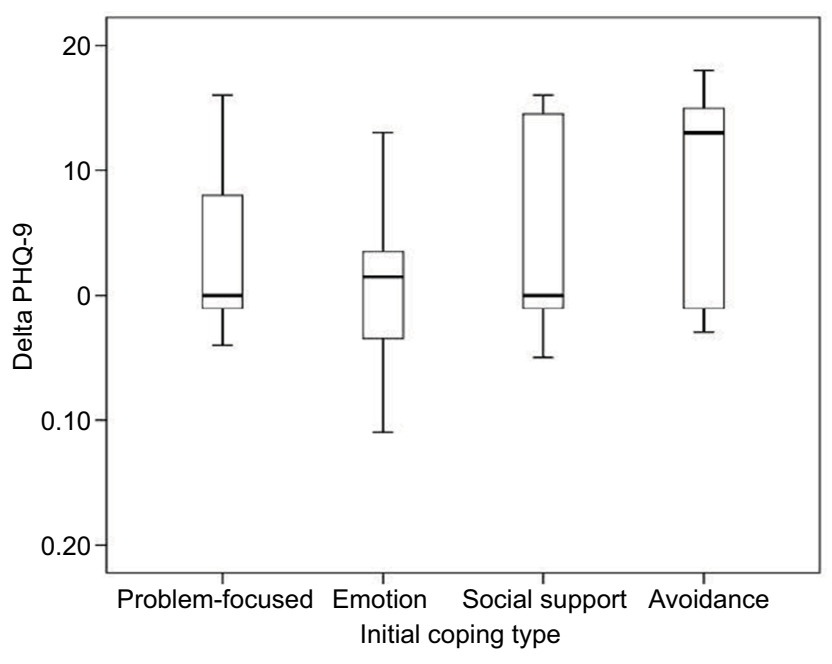

Figure 4 Variation of PHQ-9 scores according to initial patients' coping type. Abbreviation: PHQ-9, Patient Health Questionnaire-9.

(median 18 points) after receiving their diagnosis. In contrast, patients with initial emotion coping had lower GAD-7 (median 8.5 points) and PHQ-9 (median 11 points) scores after receiving the diagnosis. The detailed final GAD-7 and PHQ-9 analysis, stratified based on their initial coping, is detailed in Table 5.

We observed that there were no significant differences among the subgroups formed with respect to initial coping style regarding the initial GAD-7 $(p=0.182)$, final GAD-7 ( $p=0.465)$, initial PHQ-9 $(p=0.067)$ and final PHQ-9 scores $(p=0.276)$.

In all our patients, subgroups (stratified based on the initial coping style) of both the anxiety and depression scores were increased after diagnosis when compared to initial values. However, a set of particularities can be observed based on the initial coping styles: in patients with initial problem-focused and emotion-focused coping style, the differences between anxiety and depression scores prior versus after diagnosis were nonsignificant. The increases in the anxiety scores were significant in patients with initial social support-focused coping style ( 13 vs $3 ; p=0.014$ ) and 
avoidance coping style ( 14 vs $6 ; p=0.003$ ). Regarding the depression scores, after the diagnosis, significant increase was observed only in patients with initial avoidance coping style ( 18 vs $5 ; p=0.014$ ). The detailed results of the analysis are presented in Table 6 .

When stratified based on the final coping style, we observed that the differences in the studied scales were nonsignificant among the subgroups. This observation is valid for both initial and final moments after receiving the diagnostic, respectively, for both anxiety and depression scales.

In all the studied subgroups (stratified based on the final coping style), we observed increases in both anxiety and depression scales. However, with depression, we observed significant increases after diagnosis only in patients having social support as final coping style (16 vs $9 ; p=0.043$ ).

Table 4 Variations in GAD-7 and PHQ-9 scores after diagnosis, stratified according to initial coping type

\begin{tabular}{lll}
\hline Initial coping & $\Delta$ GAD-7 & $\Delta$ PHQ-9 \\
\hline Problem-focused & I (I2) & $0(13)$ \\
Emotion & $2(15)$ & $2(8)$ \\
Social support & $3(9)$ & $0(16)$ \\
Avoidance & $7(9)$ & $13(17)$ \\
p-value & 0.030 & 0.023 \\
\hline
\end{tabular}

Notes: Results are presented as median difference and (interquartile distance). p-values were calculated using Kruskal-Wallis test.

Abbreviations: GAD-7, Generalized Anxiety Disorder 7; PHQ-9, Patient Health Questionnaire-9.

Table 5 Depression and anxiety scores after receiving the diagnosis, stratified regarding the initial coping type

\begin{tabular}{lll}
\hline Initial coping & Final GAD-7 & Final PHQ-9 \\
\hline Problem-focused & $10(\mathrm{II})$ & $16(6)$ \\
Emotion & $8.5(10)$ & $11(12)$ \\
Social support & $13(1 \mathrm{I})$ & $14(15)$ \\
Avoidance & $14(5)$ & $18(7)$ \\
p-value & 0.465 & 0.276 \\
\hline
\end{tabular}

Notes: Results are presented as median difference and (interquartile distance). p-values were calculated using Kruskal-Wallis test.

Abbreviations: GAD-7, Generalized Anxiety Disorder 7; PHQ-9, Patient Health Questionnaire-9.
In all the studied subgroups, apart from problem-focused final coping style, the increases in the anxiety scores after diagnosis were statistically significant: emotion (11 vs 4; $p=0.021$ ), social support ( 12 vs $5 ; p=0.008$ ), and avoidance (14 vs $5 ; p=0.049$ ). This analysis is presented in Table 7.

\section{The impact of diagnosis on coping}

Of the entire cohort, 12 patients $(25.5 \%)$ had problemfocused coping, in contrast to the other 35 (74.5\%) who had another type of coping at the follow-up investigation.

In the subgroup that had initial problem-focused coping type (15 patients), 3 did not modify their coping style, another 3 migrated to emotion-focused coping, 1 to avoidance coping, and 8 (53.3\% of the entire subgroup) to social support-focused coping.

Of the patients with initial emotion-focused coping (8 patients), 2 patients did not modify their coping, 1 patient migrated to problem-focused coping, 2 patients migrated to social support-focused coping, and 3 patients migrated to avoidance-type coping.

In the cohort of patients with initial social supportfocused coping (11 patients), 3 patients had the same coping type at follow-up, 5 (45.5\% of the cohort's patients) migrated to problem-focused coping, and 3 migrated to emotion-focused coping type. No patient with initial social support-focused coping migrated to avoidance coping type.

Patients with initial avoidance coping (13 patients) were characterized by the following coping migration pattern: 4 remained in the same coping type, 3 migrated to problemfocused coping, and 6 (46.2\% of the subgroup) migrated to emotion-focused coping.

The distribution of coping styles before and 3 months after diagnosis is presented in Table 8.

\section{Discussion}

Lately, we have observed a growing interest in approaching somatic pathology in terms of psychological and psycho-

Table 6 The impact of lung cancer diagnosis on anxiety and depression stratified based on the initial coping style

\begin{tabular}{lllllll}
\hline Initial coping & Initial GAD-7 & Final GAD-7 & p-value & Initial PHQ-9 & Final PHQ-9 & P-value \\
\hline Problem-focused & $4(5)$ & $10(I I)$ & 0.063 & $12(14)$ & $16(6)$ & 0.235 \\
Emotion & $7(7)$ & $8.5(I 0)$ & 0.528 & $11(10)$ & $11(12)$ & 0.779 \\
Social support & $3(5)$ & $13(I I)$ & $0.014^{*}$ & $4(10)$ & $14(15)$ & 0.201 \\
Avoidance & $6(5)$ & $14(5)$ & $0.003^{*}$ & $5(10)$ & $18(7)$ & $0.014^{*}$ \\
p-value & 0.182 & 0.465 & & 0.067 & 0.276 & \\
\hline
\end{tabular}

Notes: *Differences are significant at $\alpha<0.05$ threshold. Results are presented as median difference and (interquartile distance). $p$-values were calculated using Kruskal-Wallis test.

Abbreviations: GAD-7, Generalized Anxiety Disorder 7; PHQ-9, Patient Health Questionnaire-9. 
Table 7 The impact of lung cancer diagnosis on anxiety and depression, stratified based on the final coping style

\begin{tabular}{lllllll}
\hline Final coping & Initial GAD-7 & Final GAD-7 & p-value & Initial PHQ-9 & Final PHQ-9 & p-value \\
\hline Problem-focused & $5(8)$ & $12(10)$ & 0.098 & $4(12)$ & $15(8)$ & 0.102 \\
Emotion & $4(7)$ & $11(12)$ & $0.021^{*}$ & $7(10)$ & $16(11)$ & 0.133 \\
Social support & $5(5)$ & $12(7)$ & $0.008^{*}$ & $9(12)$ & $16(7)$ & $0.043^{*}$ \\
Avoidance & $5(4)$ & $14(14)$ & $0.049^{*}$ & $8(11)$ & $17(16)$ & 0.309 \\
p-value & 0.839 & 0.738 & & 0.624 & 0.845 & \\
\hline
\end{tabular}

Notes: *Differences are significant at $a<0.05$ threshold. Results are presented as median difference and (interquartile distance). $p$-values were calculated using Kruskal-Wallis test.

Abbreviations: GAD-7, Generalized Anxiety Disorder 7; PHQ-9, Patient Health Questionnaire-9.

Table 8 Coping types distribution before and after diagnosis

\begin{tabular}{lll}
\hline Coping type & Initial & Follow-up (3 months) \\
\hline Problem-focused & $15(31.9 \%)$ & $12(25.5 \%)$ \\
Emotion & $8(17.0 \%)$ & $14(29.8 \%)$ \\
Social support & $11(23.4 \%)$ & $13(27.7 \%)$ \\
Avoidance & $13(27.7 \%)$ & $8(17.0 \%)$ \\
p-value & 0.344 & \\
\hline
\end{tabular}

pathology involvement, thus new studies are conducted in this direction. ${ }^{23-27}$

One of the strengths of this study was that it evaluated the coping style in patients with lung cancer in dynamics. Even after the considerable existing advancements in lung cancer treatment, there remains a threat to the patient, the risk of recurrence (post-surgery) or metastasis, constituting a major stress factor.

Research indicates that psychosocial and behavioral variables (including coping) have an important impact on the adaptation mechanism of the patients to the new situation of having a vital disease such as cancer. ${ }^{18}$

The role of coping is also well known in the management of anxiety and depression symptoms. There are extensive studies in the literature. ${ }^{28-31}$

In our study group, we observed a significant increase in anxiety and depression a month after receiving the diagnosis. Thus, these two psychiatric pathologies must be taken into account in patients with lung cancer. About $57.5 \%$ of patients had at least medium intensity anxiety and $74.4 \%$ at least medium depression a month after receiving the diagnosis.

Receiving the diagnosis of lung cancer has a significant impact on the psychic level, with half of the patients developing depression and anxiety that needs psychiatric approach. This aspect is in accordance with the literature that mentions a frequent association of these types of comorbidities in patients with lung cancer. ${ }^{6-8,32}$

The greatest increase in anxiety intensity was found in patients with avoidance coping followed by those with social support-focused coping. This would suggest that patients with these types of coping have the lowest efficiency in managing secondary anxiety after receiving the news. We also found a statistically significant increase in depression in patients with avoidance coping compared with other groups. No significant increase was found in the other groups regarding depression. We can conclude that patients with initial avoidance coping were the least efficient in managing traumatizing situations, whereas patients with emotional coping had the best management in this situation.

After receiving the diagnosis and changing coping styles, only those with problem-focused coping after 1 month had no significant increase in anxiety and depression. Those with other coping styles had eventually a significant increase in anxiety and the social support style had an increase in depression.

We mention that among the four subgroups, no significant differences in the intensity of depression and anxiety were recorded; neither at the beginning of the study nor at the end after regrouping the patients according to their new migrated coping styles. The majority of the subjects modified their coping styles to manage the situation created by the existence of a terminal disease.

It is interesting to note that patients who initially had an emotional coping style, with the highest values of anxiety, had the lowest increase in anxiety and depression after receiving the diagnosis. Thus, emotional coping style could be considered adaptive for patients with lung cancer. If we consider the emotional style adaptive, besides the well-known adaptive role of the problem-focused style, we consider that psychological intervention conducive to the development of both the styles is useful in these types of patients.

In a study that included 80 women with breast cancer, Cohen observed that emotion-focused coping could specifically help the patients reduce the level of anxiety. ${ }^{33}$

The least used coping style was the avoidant one $(17 \%$ of subjects). This would suggest that the majority of the patients mobilized a coping style that requires certain activation. This aspect corresponds with Foley et al's observations. Their study included 58 cancer patients who were diagnosed 
5 years earlier and had been treated for a variety of cancer types. The majority of the patients $(65.5 \%)$ accepted the fact that they had cancer and chose to go on with bravery (willing). About $21 \%$ of these patients tried to manage the event by considering it an opportunity for growth. This aspect was more frequent in young adults and women. ${ }^{34}$

The mobilization of the patients with cancer in the early stages is seen as a good strategy but late stages of the disease represent no viable option. Thus, if in the initial stages of the disease, active coping style was found to be associated with lower distress, the situation could change with the disease progression. This could be explained through the initial sensation of "being in control" in the initial stages of the disease by adopting a more active coping style. But when the disease reaches a point where no therapeutic intervention can be useful, such type of coping becomes less beneficial and a more accepting strategy can become more helpful. ${ }^{35}$

\section{Conclusion}

The study results are pointing to a significant increase in the intensity of both depression and anxiety after receiving the diagnosis of lung cancer. A large proportion of patients tend to modify their coping styles, thus the coping mechanisms used 1 month after diagnosis versus baseline are, in many cases, different.

The most adaptive coping style turned out to be the problem-focused style while the least adaptive was the avoidance-coping style.

\section{Acknowledgment}

The research study was supported by grant Innovative Programme III-C2-PCFI-2015/2016 (partnerships in fundamental research).

\section{Disclosure}

The authors report no conflicts of interest in this work.

\section{References}

1. Social styrelsen [National Board of Health and Welfare]. Epidemiologiskt centrum, 2004 [Epidemiologist Center, 2004]. Available from: http:// www.socialstyrelsen.se/NR/rdonlyres/A6DB1C7C-D106-4413-B8DBC7C3F3229542/5464/2006423.pdf. Accessed February 1, 2017. Polish.

2. Jemal A, Bray F, Center MM, Ferlay J, Ward E, Forman D. Global cancer statistics. CA Cancer J Clin. 2011;61:69-90.

3. Cancer facts and figures. Available from: www.cancer.org. Accessed 17.10.2017

4. Carlson LE, Bultz BD. Cancer distress screening: needs, models and methods. J Psychosom Res. 2003;55(5):403-409.

5. Vachon M. Psychosocial distress and coping after cancer treatment. Cancer Nurs. 2006;29(2):26-31.
6. Zabora J, Brintzenhofeszoc K, Curbow B, Hooker C, Piantadosi S. The prevalence of psychological distress by cancer site. Psychooncology. 2001;10(1):19-28.

7. Gore JM, Brophy CJ, Greenstone MA. How well do we care for patients with end stage chronic obstructive pulmonary disease (COPD)? A comparison of palliative care and quality of life in COPD and lung cancer. Thorax. 2000;55:1000-1006.

8. Carmack Taylor CL, Badr H, Lee JH, et al. Lung cancer patients and their spouses: psychological and relationship functioning within 1 month of treatment initiation. Ann Behav Med. 2008;36:129-140.

9. Carlson LE, Bultz BD. Cancer distress screening: needs, models, and methods. J Psychosom Res. 2003;55(5):403-409.

10. Kuo TT, Ma FC. Symptom distress and coping strategies in patients with non-small cell lung cancer. Cancer Nurs. 2002;25:309-317.

11. Henoch I, Bergman, B, Gustafsson, M., et al. The impact of symptoms, coping capacity, and social support on quality of life experience over time in patients with lung cancer. $J$ Pain Symptom Manage. 2007;34(4):370-379.

12. Rand KL, Cripe LD, Monahan PO. Illness appraisal, religious coping, and psychological responses in men with advanced cancer. Support Care Cancer. 2012;20:1719-1728.

13. Akechi T, Kugaya A, Okamura H, Nishiwaki Y, Yamawaki S, Uchitomi Y. Predictive factors of psychological distress in ambulatory lung cancer patients. Support Care Cancer 1998;6:281-286.

14. Walker MS, Zona DM, Fisher EB. Depressive symptoms after lung cancer surgery: their relation to coping style and social support. Psychooncology. 2006;15(8):684-693.

15. Mosher CE, Ott MA, Hanna N, et al. Coping with physical and psychological symptoms: a qualitative study of advanced lung cancer patients and their family caregivers. Support Care Cancer. 2015;23(7):2053-2060.

16. Lechner SC, Zakowski SG, Antoni MH, et al. Do sociodemographic and disease-related variables influence benefit-finding in cancer patients? Psychooncology. 2003;12(5):491-499.

17. Spencer SM, Carver CS, Price AA. Psychological and social factors in adaptation. In: Holland JC, editor. Psycho-oncology. New York: Oxford University Press; 1998:211-222.

18. Boehmer S, Luszczynska A, Schwarzer R. Coping and quality of life after tumor surgery: personal and social resources promote different domains of quality of life. Anxiety Stress Coping. 2007;20(1):61-75.

19. Carver CS, Scheier MF, Weintraub JK. Assessing coping strategies: a theoretically based approach. J Personal Soc Psychol. 1989;56:267-283.

20. Crasovan DI, Sava FA. Translation, adaptation and validation on Romanian population of COPE questionnaire for coping mechanisms analysis. Cogn Brain Behav. 2013;17(1):61-76.

21. Spitzer RL, Kroenke K, Williams JB; Patient Health Questionnaire Study Group. Validity and utility of a self-report version of PRIMEMD: the PHQ Primary Care Study. JAMA. 1999;282(18):1737-1744.

22. Spitzer RL, Kroenke K, Williams JB, Löwe B. A brief measure for assessing generalized anxiety disorder: the GAD-7. Arch Intern Med. 2006;166(10):1092-1097.

23. Enatescu VR, Papava I, Enatescu I, et al. Circulating plasma micro RNAs in patients with major depressive disorder treated with antidepressants: a pilot study. Psychiatry Investig. 2016;13(5):549-557.

24. Enatescu VR, Enatescu I, Craina M, et al. State and trait anxiety as a psychopathological phenomenon correlated with postpartum depression in a Romanian sample: a pilot study. J Psychosom Obstet Gynaecol. 2014;35(2):55-61.

25. Mut-Vitcu G, Timar B, Timar R, Oancea C, Citu IC. Depression influences the quality of diabetes-related self-management activities in elderly patients with type 2 diabetes: a cross-sectional study. Clin Interv Aging. 2016;11:471-479.

26. Crişan AF, Oancea C, Timar B, Fira-Mladinescu O, Crişan A, Tudorache $\mathrm{V}$. Cognitive impairment in chronic obstructive pulmonary disease. PLoS One. 2014;9(7):e102468. 
27. Papava I, Oancea C, Enatescu VR, et al. The impact of coping on the somatic and mental status of patients with COPD: a cross-sectional study. Int J Chron Obstruct Pulmon Dis. 2016;11:1343-1351.

28. Crockett LJ, Iturbide MI, Torres Stone, McGinley M, Raffaelli M, Carlo G. Acculturative stress, social support, and coping: relations to psychological adjustment among Mexican American college students. Cultur Divers Ethnic Minor Psychol. 2007;13(4):347-355.

29. Penland E, Masten W, Zelhart P, Fournet GP, Callahan TA. Possible selves, depression, and coping skills in university students. J Personal Individ Differ. 2000;29:963-969.

30. Sherbourne C, Hays RD, Wells KB. Personal and psychosocial risk factors for physical and mental health outcomes and course of depression among depressed patients. J Consult Clin Psychol. 1995;63(3): 345-355.
31. Knibb RC, Horton SL. Can illness perceptions and coping predict psychological distress amongst allergy sufferers? Br J Health Psychol. 2008;13(1):103-119.

32. Dudgeon DJ, Kristjanson L, Sloan JA, Lertzman M, Clement K. Dyspnea in cancer patients: prevalence and associated factors. J Pain Symptom Manage. 2001;21(2):95-102.

33. Cohen M. Coping and emotional distress in primary and recurrent breast cancer patients. J Clin Psychol Med Settings. 2002;9(3):245-251.

34. Foley KL, Farmer DF, Petronis VM, et al. A qualitative exploration of the cancer experience among long-term survivors: comparisons by cancer type, ethnicity, gender, and age. Psychooncology. 2006;15:248-258.

35. Voogt E, van der HA, Rietjens JA, et al. Attitudes of patients with incurable cancer toward medical treatment in the last phase of life. J Clin Oncol. 2005;23:2012-2019.
Cancer Management and Research

\section{Publish your work in this journal}

Cancer Management and Research is an international, peer-reviewed open access journal focusing on cancer research and the optimal use of preventative and integrated treatment interventions to achieve improved outcomes, enhanced survival and quality of life for the cancer patient. The manuscript management system is completely online and includes
Dovepress

a very quick and fair peer-review system, which is all easy to use. Visit $\mathrm{http}: / / \mathrm{www}$.dovepress.com/testimonials.php to read real quotes from published authors. 